\title{
Manipulating the torsion of molecules by strong laser pulses
}

\author{
C.B. Madsen, ${ }^{1}$ L.B. Madsen,,${ }^{1}$ |ศ S.S. Viftrup,${ }^{2}$ M. P. Johansson,${ }^{2}$ T.B. Poulsen, ${ }^{2}$ \\ L. Holmegaard, ${ }^{2}$ V. Kumarappan, ${ }^{2}$ K.A. Jørgensen, ${ }^{2}$ and H. Stapelfeldt ${ }^{3, \dagger}$ \\ ${ }^{1}$ Lundbeck Foundation Theoretical Center for Quantum System Research, \\ Department of Physics and Astronomy, Aarhus University, 8000 Aarhus C, Denmark \\ ${ }^{2}$ Department of Chemistry, Aarhus University, 8000 Aarhus C, Denmark \\ ${ }^{3}$ Department of Chemistry and Interdisciplinary Nanoscience Center (iNANO), Aarhus University, 8000 Aarhus C, Denmark
}

(Dated: August 1, 2021)

\begin{abstract}
A proof-of-principle experiment is reported, where torsional motion of a molecule, consisting of a pair of phenyl rings, is induced by strong laser pulses. A nanosecond laser pulse spatially aligns the carbon-carbon bond axis, connecting the two phenyl rings, allowing a perpendicularly polarized, intense femtosecond pulse to initiate torsional motion accompanied by an overall rotation about the fixed axis. The induced motion is monitored by femtosecond time-resolved Coulomb explosion imaging. Our theoretical analysis accounts for and generalizes the experimental findings.
\end{abstract}

PACS numbers: $33.15 . \mathrm{Hp}, 33.80 . \mathrm{Rv}, 42.50 . \mathrm{Hz}$

A non-resonant laser field applies forces and torques on molecules due to the interaction between the induced dipole moment and the laser field itself. If the field is intense, but non-ionizing, the forces and torques can be sufficient to effectively manipulate the external degrees of freedom of isolated gas phase molecules. In particular, the intensity gradient of a focused laser beam may deflect [1], focus [2] and slow 3 molecules through the dependence of the non-resonant polarizability interaction on the intensity. Likewise, the dependence of the induced dipole interaction on molecular orientation has proven highly useful for controlling the alignment and rotation of a variety of molecules [4, 5]. Molecular manipulation by induced dipole forces extends beyond the external degrees of freedom and can also be applied to the internal degrees of freedom such as vibrational motion [6]. Notably, the electrical field from laser pulses can modify energy potential barriers such that photoinduced bond breakage of a small linear molecule is guided to yield a desired final product [7, 8].

Here, we extend the use of the non-resonant polarizability interaction to achieve a transient modification of the torsional potential of the molecule 3,5-diflouro3',5'-dibromo-biphenyl (DFDBrBPh), thereby inducing torsional motion of the two phenyl rings (Fig. 1). The results are accounted for by our theoretical analysis, and we point at two very diverse aspects of the current work: (i) The potential application of twisted molecules in, e.g., molecular junctions, where they may serve as ultrafast (picosecond) molecular switches [9, 10, 11, 12. (ii) DFD$\mathrm{BrBPh}$ has two conformations that are mirror images of each other, the $\mathrm{R}_{a}$ and $\mathrm{S}_{a}$ enantiomers [13]. An extension of the present experiment will provide a unique temporally resolved study of the important chemical process de-racemization [14, 15, 16, 17, where one enantiomer is selectively converted into the other.

The experiment is carried out on isolated molecules at rotational temperatures of a few Kelvin. Under these (a)

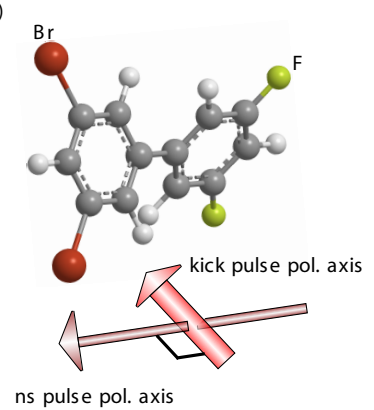

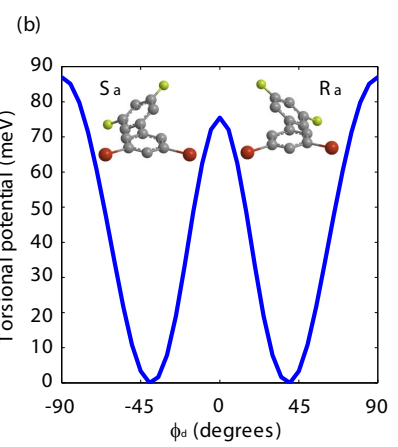

FIG. 1: (Color online) (a) Model of DFDBrBPh and the laser geometry of the experiment. The molecule consists of a pair of phenyl rings. The substituted $\mathrm{Br}$ and $\mathrm{F}$ atoms are needed to discriminate the two rings in the experiment. (b) The torsional potential of DFDBrBPh [18. The torsion is quantified by the dihedral angle, $\phi_{d}$, between the two phenyl rings. The minima at dihedral angles of $\phi_{d}= \pm 39^{\circ}$ result in the $\mathrm{R}_{a}$ and $\mathrm{S}_{a}$ enantimomers (see text).

conditions each molecule is initially localized in the $-39^{\circ}$ or $39^{\circ}$ conformation (Fig. $1 \mathrm{~b}$ ) and no thermally induced transitions between the two occur. The scheme to obtain torsional motion is based on the combination of two laser beams (Fig. 1 1a). First, a 1064 nm, 9 ns (FWHM) linearly polarized laser pulse of peak intensity $7 \times 10^{11}$ $\mathrm{W} / \mathrm{cm}^{2}$ fixes the carbon-carbon (C-C) axis between the two phenyl rings in the laboratory frame by adiabatic alignment [4, 19]. This pulse is intense enough to keep the axis tightly confined, yet weak enough to modify the torsional potential only slightly. Next, an $800 \mathrm{~nm}$ laser pulse of intensity $5 \times 10^{12} \mathrm{~W} / \mathrm{cm}^{2}$ and duration (FWHM) 700 fs, which we will refer to as the kick pulse, is applied with its polarization perpendicular to the aligned $\mathrm{C}-\mathrm{C}$ bond axis. This polarization geometry ensures that the kick pulse primarily influences torsional motion while avoiding excitation of other normal modes. At time $t_{p}$ with 

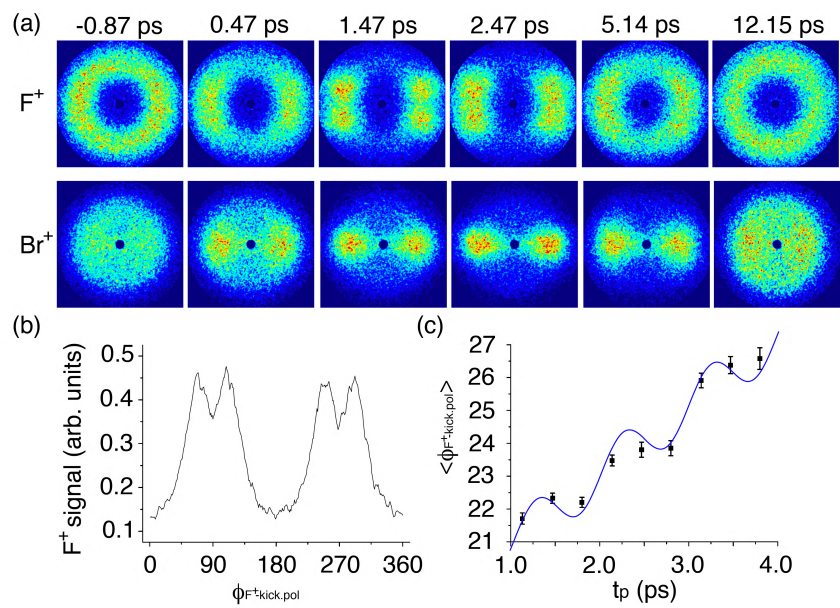

(c)

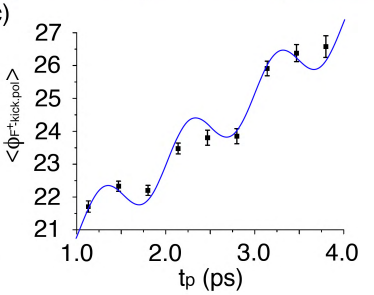

FIG. 2: (Color online) (a) Ion images of $\mathrm{F}^{+}$and $\mathrm{Br}^{+}$fragments at probe times $t_{p}$. The ns pulse is polarized perpendicularly to the image (detector) plane and the $5 \times 10^{12} \mathrm{~W} / \mathrm{cm}^{2}$, $700 \mathrm{fs}$ (FWHM) kick pulse is polarized horizontally. (b) Angular distribution of the $\mathrm{F}^{+}$ions, at $t_{p}=1.47 \mathrm{ps}$, obtained by radially integrating the corresponding $\mathrm{F}^{+}$ion image between the $\mathrm{F}^{+}$ion recoil and the kick pulse polarization for fixed angle, $\phi_{\mathrm{F}+\text { - kick. pol }}$. The splitting of the pairwise peaks is twice the the average angle, $\left\langle\phi_{\mathrm{F}^{+} \text {- kick. pol }}\right\rangle$, between the $\mathrm{F}^{+}$ion recoil and the kick pulse polarization. (c) $\left\langle\phi_{\mathrm{F}+\text { - kick. pol }}\right\rangle$ as a function of $t_{p}$, for times where a clear four-peak structure is visible in the angular distributions. The curve is a fit of the sum of a linear and a harmonic function to the experimental points (squares).

respect to the kick pulse, a linearly polarized, intense 800 $\mathrm{nm}, 25 \mathrm{fs}(\mathrm{FWHM}), 2 \times 10^{14} \mathrm{~W} / \mathrm{cm}^{2}$ pulse appears and removes several electrons from the molecules, thereby triggering Coulomb explosion into ionic fragments. In particular, the $\mathrm{Br}^{+}$and $\mathrm{F}^{+}$fragment ions recoil in the planes defined by the two phenyl rings. By recording the velocities of both ion species with two-dimensional ion imaging [19], we thus determine the instantaneous orientation of each of the two phenyl rings at $t_{p}$. The time-resolved ion images are displayed in Fig. 2a.

To establish that the ns pulse aligns the $\mathrm{C}-\mathrm{C}$ axis, we have applied the probe pulse at $t_{p}=-0.87 \mathrm{ps}$. This results in almost circularly symmetric ion images (Fig. 2a) and the small deviation from circular symmetry is explained by noting that the kick pulse has a finite value at $-0.87 \mathrm{ps}$. The absence of ions in the innermost region, most clearly seen in the $\mathrm{F}^{+}$image, shows that the $\mathrm{C}-\mathrm{C}$ bond is aligned perpendicular to the detector plane and that the rotation of the molecule around this axis is uniform. At $t_{p}=0.47 \mathrm{ps}$ the deviation from circular symmetry is very clear and the ions start to localize around the polarization direction of the kick pulse. The $\mathrm{F}^{+}$ions remain radially confined away from the center, which shows that the kick pulse does not perturb the alignment of the $\mathrm{C}-\mathrm{C}$ bond axis. Rather, it initiates an overall rotation of the molecule around this axis as is ex- pected since the torque imparted by the kick pulse forces the second most polarizable axis (SMPA), perpendicular to the $\mathrm{C}-\mathrm{C}$ axis and located $11^{\circ}$ away from the Br-phenyl ring, to align along the kick pulse polarization on a time scale determined by the kick strength [20. At $t_{p}=1.47$ ps the $\mathrm{Br}^{+}$ions are localized around the polarization axis and the $\mathrm{F}^{+}$ion distribution exhibits a four-peaked structure. This behavior is compatible with alignment of the SMPA along the kick pulse polarization. In practice and consistent with theory (Fig. 3a), the SMPA alignment is not strong enough to resolve the two $\mathrm{Br}^{+}$signals located at $\pm 11^{\circ}$ with respect to the SMPA. It is, however, sharp enough to resolve the two pairs of $\mathrm{F}^{+}$ion signals due to the larger offset $\left(28^{\circ}\right)$ of the F-phenyl rings from the SMPA. The further localization of the $\mathrm{Br}^{+}$signal at 2.47 ps shows that the Br-phenyl planes have rotated into stronger alignment with the kick pulse polarization. Had the dihedral angle remained unchanged, the $\mathrm{F}^{+}$ion image should exhibit a distinct four-dot structure similar to the image at $1.47 \mathrm{ps}$ but with a larger angular splitting. The four-dot structure at $2.47 \mathrm{ps}$ is, however, significantly blurred compared to the case at 1.47 ps. Thus, we conclude that the kick pulse sets the molecule into rotation around the $\mathrm{C}-\mathrm{C}$ axis and initiates torsional motion. At later times, the ion signals gradually broaden due to continued overall rotation around the $\mathrm{C}-\mathrm{C}$ axis with dihedral dynamics imposed.

Further insight into the effect of the kick pulse is obtained by plotting the average angle between the Fphenyl rings and the kick pulse polarization as a function of $t_{p}$ (Fig. 2c). The increase from $22.5^{\circ}$ at 1.47 ps to $26.5^{\circ}$ at 3.8 ps shows that the F-phenyl rings gradually move away from the kick pulse polarization due to the overall rotation of the molecule around the $\mathrm{C}-\mathrm{C}$ axis. The concurrent oscillations show that the overall rotation is accompanied by a periodically varying motion in $\phi_{d}$. We estimate the period to be $\sim 1$ ps and the amplitude to $\sim 0.6^{\circ}$ for this oscillation.

We now present our physical model substantiating the experimental findings. In agreement with the observations, we assume that the $\mathrm{C}-\mathrm{C}$ axis is perfectly aligned. Also, we neglect all normal modes, except the lowest one, corresponding to torsion. Within these approximations the task is reduced to describing the coupled rotations of the two phenyl rings. The experimental observables are the angles $\phi_{\mathrm{Br}}$ and $\phi_{\mathrm{F}}$ of the rings with respect to the kick pulse polarization axis. In the theoretical treatment it is, however, convenient to refer to the dihedral angle $\phi_{d}=\phi_{\mathrm{Br}}-\phi_{\mathrm{F}}$ and the overall rotation around the $\mathrm{C}-\mathrm{C}$ axis described by the weighted azimuthal angle $\Phi=(1-\eta) \phi_{\mathrm{Br}}+\eta \phi_{F}$, with $\eta$ given in terms of the moments of inertia, $\eta=I_{\mathrm{F}} /\left(I_{\mathrm{Br}}+I_{\mathrm{F}}\right)$. This change of coordinates separates the dynamics of the molecule into two rotations of different time scales. The first, $\phi_{d}$, is an internal rotation corresponding to torsional motion. The time scale of torsion is ps, as can 


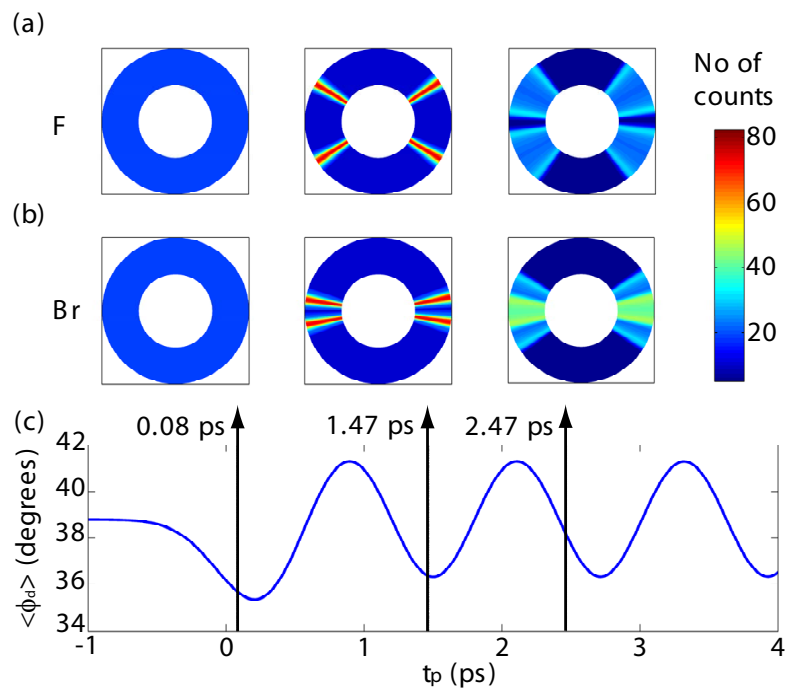

FIG. 3: (Color online) Angular distributions of (a) F-phenyl and (b) Br-phenyl rings at $t_{p}=0.08,1.47$ and 2.47 ps. (c) Expectation value of the dihedral angle for a molecule starting out with the SMPA aligned along the kick pulse polarization. The kick pulse is as in Fig. 2.

be inferred from the torsional potential. The second rotation, $\Phi$, is an overall rotation with a period of ns, as follows from the total moment of inertia $I=I_{\mathrm{Br}}+I_{\mathrm{F}}$. This separation motivates the introduction of a semiclassical model, where $\phi_{d}$ is treated fully quantum mechanically, while $\Phi$ is treated classically. Briefly, due to the kick pulse the field-free torsional state of energy $E_{\nu}$ evolves as [we apply atomic units, $m_{e}=e=a_{0}=$ $\hbar=1]\left|\chi_{\nu}\right\rangle \rightarrow\left|\chi_{\nu}^{\Phi}(t)\right\rangle=\sum_{\nu^{\prime}} c_{\nu^{\prime}}^{\Phi}(t) e^{-i E_{\nu^{\prime}}\left(t-t_{0}\right)}\left|\chi_{\nu^{\prime}}\right\rangle$, with $t_{0}$ a time prior to the kick pulse. The timedependent coefficients satisfy the differential equations $\dot{c}_{\nu^{\prime}}^{\Phi}(t)=-i \sum_{\nu} c_{\nu}^{\Phi}(t) e^{-i\left(E_{\nu}-E_{\nu^{\prime}}\right)\left(t-t_{0}\right)}\left\langle\chi_{\nu^{\prime}}\left|V_{\text {kick }}(\Phi, t)\right| \chi_{\nu}\right\rangle$, with the kick pulse polarization interaction

$$
\begin{aligned}
V_{\mathrm{kick}}\left(\Phi, \phi_{d}, t\right) & =-\frac{1}{4} F_{0}^{2}(t)\left[\alpha_{\mathrm{xx}}\left(\phi_{d}\right) \cos ^{2}\left(\Phi+\eta \phi_{d}\right)\right. \\
& +\alpha_{\mathrm{yy}}\left(\phi_{d}\right) \sin ^{2}\left(\Phi+\eta \phi_{d}\right) \\
& \left.-2 \alpha_{\mathrm{xy}}\left(\phi_{d}\right) \cos \left(\Phi+\eta \phi_{d}\right) \sin \left(\Phi+\eta \phi_{d}\right)\right] .
\end{aligned}
$$

Here $\alpha_{i j}$ 's are dynamic polarizability tensor components obtained by our quantum chemistry calculations and $F_{0}$ is the kick pulse field envelope. The effect of the kick pulse on the overall rotation amounts to an angular momentum kick and consequently

$$
\Phi(t)=\Phi_{0}-\left.t \frac{1}{I}\left(\frac{\partial}{\partial \Phi} \int_{-\infty}^{\infty} d t^{\prime}\left\langle V_{\text {kick }}\left(\Phi, t^{\prime}\right)\right\rangle\right)\right|_{\Phi=\Phi_{0}},
$$

where \langle\rangle denotes averaging over the ensemble of torsional states.

Figure 3 shows the results of a calculation with laser parameters identical to the experimental values and an initial rotational temperature of $0 \mathrm{~K}$. Prior to the kick pulse the angular distributions of the Br- and F-phenyl rings (left panels, Figs. $3 \mathrm{a}$ and $3 \mathrm{~b}$ ) are isotropic as in the experiment. Maximum alignment of the SMPA occurs at 1.3 ps and the confinement of the F-phenyl rings at a large angle with respect to the kick pulse polarization (cf. middle panel, Fig. 3a) explains the distinct four-dot structure observed at $t_{p}=1.47$ ps (Fig. 2a) in the experimental $\mathrm{F}^{+}$ion image. Also, at $t_{p}=1.47 \mathrm{ps}$ the confinement of the Br-phenyl rings at a small angle with respect to the kick pulse polarization predicts a much less distinct, if any, four-dot structure in good agreement with the $\mathrm{Br}^{+}$ion image. At $t_{p}=2.47$ ps the angular localization of the F-phenyl rings has broadened (right panel, Fig. 3a) and a blurred four-dot structure is seen, consistent with the experimental result at $t_{p}=2.47 \mathrm{ps}$. The distribution of the Br-phenyl rings is also broadened (right panel, Fig. 3b) but remains localized around the kick pulse polarization fully consistent with the $\mathrm{Br}^{+}$ion distribution, recorded at $2.47 \mathrm{ps}$.

The theoretical value $\left\langle\phi_{d}\right\rangle$ exhibits oscillations (Fig. 3c) with a period of $\sim 1.2$ ps and amplitude of $\sim 2.45^{\circ}$. The period agrees well with the experimental value $(\sim 1 \mathrm{ps})$, and smaller modulation in $\left\langle\phi_{d}\right\rangle$ is expected in the experiment $\left(\sim 0.6^{\circ}\right)$ since here the SMPA is not pre-aligned. The behavior is ascribed to a wave packet of vibrational modes in the torsional double well potential (Fig. 1 b) for a molecule starting out with the SMPA aligned. The qualitative agreement of Figs. 2b and $3 \mathrm{c}$ verifies the interpretation of the kick pulse inducing time-dependent torsional motion.

Now we point at some possible future applications of induced torsional motion. For one thing, DFDBrBPh represents a class of molecules, where the conductivity can be controlled by manipulating the torsion. Such a molecule used as a molecular junction between two conductors thus offers an attractive alternative to mechanical break junctions 21]. In particular, the fact that the dihedral angle with the present laser pulse-based method can be altered on a ps time scale opens intriguing possibilities for studying ultrafast modulation and switching of electrical charge flow.

Another new application within reach is a timeresolved study of de-racemization [14, 15, 16, 17], where one enantiomer is steered into its mirror form. To this end, we break the inversion symmetry with respect to the $\mathrm{C}-\mathrm{C}$ bond axis by orienting each molecule rather than just aligning in order to discriminate between the two enantiomeric forms. Theory [22] and experiment 23] show that orientation can be added to $3 \mathrm{D}$ alignment by combining the ns alignment pulse with a static electric field. Next, to reduce the role of overall rotation, the SMPA can be aligned prior to the kick pulse by employing an elliptically rather than a linearly polarized ns pulse 4, 12. Finally, the interaction strength between the molecule and the kick pulse needs to be increased either through higher intensity, a longer kick pulse or by 


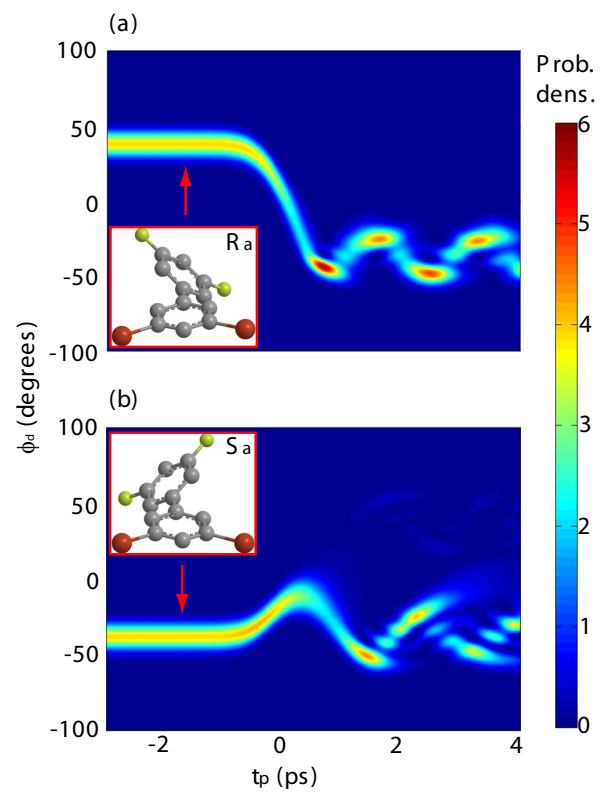

FIG. 4: (Color online) Time evolution of the dihedral angle for a molecule starting out as (a) an $\mathrm{R}_{a}$ or (b) an $\mathrm{S}_{a}$ enantiomer. Initially, the molecule is $3 \mathrm{D}$ oriented with the $\mathrm{Br}-$ phenyl end pointing out of the paper and the SMPA aligned at an angle of $13^{\circ}$ with respect to the kick pulse polarization. The kick pulse triggering the torsional motion has a peak intensity of $1.2 \times 10^{13} \mathrm{~W} / \mathrm{cm}^{2}$ and duration (FWHM) of $1.0 \mathrm{ps}$. The torsional barrier is reduced by $1 / 4$ rather than increasing the kick strength. Such modification of the torsional potential may be accomplished by replacing DFDBrBPh with, e.g., halogen substituted biphenylacetylene.

trains of synchronized kick pulses [24, 25, 26]. Assuming initial orientation and confinement of the SMPA, we have calculated the $\phi_{d}$ dynamics for both conformations of a molecule closely related to DFDBrBPh. The results are shown in Fig. 4, and clearly, the present method would allow for a time-resolved study of the transition from one enantiomer and into the other. A quantitative analysis of the efficiency of the process shows that after the pulse $99 \%$ of the molecules starting out as $\mathrm{R}_{a}$ changed into $\mathrm{S}_{a}$ enantiomers, whereas only $13 \%$ of the $\mathrm{S}_{a}$ enantiomers changed into $\mathrm{R}_{a}$. The inverse process causing an excess of $\mathrm{R}_{a}$ enantiomers, is simply achieved by inverting the orientation of the molecules.

In conclusion, we have performed fs time-resolved studies of torsional motion by a combination of aligning, kick and ionizing pulses, and we supported the observations by an accompanying theory. We believe that the present work opens new directions of cross-disciplinary research firmly anchored in strong-field physics as exemplified by ultrafast swithing in molecular junctions and fs timeresolved studies of de-racemization.

The work was supported by the Danish Research Agency, the Danish National Research Foundation and the Carlsberg Foundation.

* Corresponding author: bojer@phys.au.dk

$\dagger$ Corresponding author: henriks@chem.au.dk

[1] H. Stapelfeldt, H. Sakai, E. Constant, and P. B. Corkum, Phys. Rev. Lett. 79, 2787 (1997).

[2] H.S. Chung, B.S. Zhao, S.H. Lee, S. G. Hwang, K. C. Cho, S. H. Shim, S. W. K. Kang, and D. S. Chung, J. Chem. Phys. 114, 8293 (2001).

[3] R. Fulton, A. Bishop, M. Shneider, and P. Barker, Nature Phys. 2, 465 (2006), ISSN 1745-2473.

[4] H. Stapelfeldt and T. Seideman, Rev. Mod. Phys. 75, 543 (2003).

[5] D. Villeneuve, S.A. Aseyev, P. Dietrich, M. Spanner, M. Yu Ivanov, and P. B. Corkum, Phys. Rev. Lett. 85, 542 (2000).

[6] H. Niikura, P. Corkum, and D. Villeneuve, Phys. Rev. Lett. 90, 203601 (2003).

[7] B. Sussman, D. Townsend, M. Ivanov, and A. Stolow, Science 314, 278 (2006).

[8] B. Sussman, J. Underwood, R. Lausten, M. Ivanov, and A. Stolow, Phys. Rev. A 73, 053403 (2006).

[9] A. Nitzan and M. A. Ratner, Science 300, 1384 (2003).

[10] Z. J. Donhauser et al., Science 292, 2303 (2003).

[11] J. Chen and M. Reed, Chem. Phys. 281, 127 (2002).

[12] S. Ramakrishna and T. Seideman, Phys. Rev. Lett. 99, 103001 (2007).

[13] E. L. Eliel and S. H. Wilen, Stereochemistry of Organic Compounds (Wiley, New York, 1994), chapter 14.

[14] K. Faber, Chem. Eur. J. 7, 5004 (2001).

[15] Y. Fujimura, L. Gonzalez, K. Hoki, J. Manz, and Y. Ohtsuki, Chem. Phys. Lett. 306, 1 (1999).

[16] M. Shapiro, E. Frishman, and P. Brumer, Phys. Rev. Lett. 84, 1669 (2000).

[17] D. Kröner and B. Klaumünzer, Chem. Phys. 338, 268 (2007).

[18] M. P. Johansson and J. Olsen, J. Chem. Theory Comput. 4, 1460 (2008).

[19] V. Kumarappan, C. Z. Bisgaard, S. S. Viftrup, L. Holmegaard, and H. Stapelfeldt, J. Chem. Phys. 125, 194309 (2006).

[20] S. Viftrup, V. Kumarappan, S. Trippel, H. Stapelfeldt, E. Hamilton, and T. Seideman, Phys. Rev. Lett. 99, 143602 (2007).

[21] S. Wu, M. T. Gonźalex, R. Huber, S. Grunder, M. Mayor, C. Schönenberger, and M. Calame, Nature Nanotech. 569, 3 (2008).

[22] B. Friedrich and D. Herschbach, J. Phys. Chem. A 103, 10280 (1999).

[23] H. Tanji, S. Minemoto, and H. Sakai, Phys. Rev. A 72, 063401 (2005).

[24] M. Leibscher, I. Sh. Averbukh, and H. Rabitz, Phys. Rev. Lett. 90, 213001 (2003).

[25] C. Z. Bisgaard, M. D. Poulsen, E. Péronne, S. S. Viftrup, and H. Stapelfeldt, Phys. Rev. Lett. 92, 173004 (2004).

[26] K. F. Lee, I. V. Litvinyuk, P. W. Dooley, M. Spanner, D. M. Villeneuve, and P. B. Corkum, J. Phys. B 37, L43 (2004). 